\title{
New Bounds for Gauss Sums Derived From $k$-th Powers, and for Heilbronn's Exponential Sum
}

\author{
D.R. Heath-Brown \\ Magdalen College, Oxford \\ S. Konyagin \\ Moscow State University
}

\section{Introduction}

This paper is concerned with the Gauss sums

$$
G(a)=G_{p}(a, k)=\sum_{n=1}^{p} e_{p}\left(a n^{k}\right)
$$

and with Heilbronn's sum

$$
H(a)=H_{p}(a)=\sum_{n=1}^{p} e\left(\frac{a n^{p}}{p^{2}}\right),
$$

where $p$ is prime, $e(x)=\exp (2 \pi i x)$, and $e_{p}(x)=e(x / p)$. In each case we shall assume that $p \nmid a$ unless the contrary is explicitly stated.

Gauss sums arise in investigations into Waring's problem, and other additive problems involving $k$-th powers. Although they are amongst the simplest complete exponential sums, the question as to their true order of magnitude is far from being resolved. We remark at the outset that if $(k, p-1)=k_{0}$, then

$$
G_{p}(a, k)=G_{p}\left(a, k_{0}\right) .
$$

Thus it suffices to suppose, as indeed we shall, that $k \mid p-1$.

When $p \nmid a$ the trivial bound for $G(a)$ states that $|G(a)| \leq p$. The next simplest estimate takes the form

$$
|G(a)| \leq(k-1) \sqrt{p} .
$$

This may be obtained by writing $G(a)$ in terms of the character Gauss sum as

$$
G(a)=\sum_{\substack{\chi^{k}=\chi_{0} \\ \chi \neq \chi_{0}}} \bar{\chi}(a) \tau(\chi) .
$$


There are $k-1$ terms here, each of modulus $\sqrt{p}$. One can also think of the estimate (1) as deriving from Weil's Riemann Hypothesis for curves over finite fields. The formula (2) then gives explicitly the decomposition of $G(a)$ as a linear combination of roots of the corresponding $L$-function. We should remark that Montgomery, Vaughan and Wooley [5] have given a small improvement on (1), by showing that if $2 k \chi(p-1)$ then

$$
|G(a)| \leq 2^{-1 / 2}\left(k^{2}-2 k+2\right)^{1 / 2} p^{1 / 2},
$$

for $p>2$. Moreover they present both numerical and heuristic evidence in support of the conjecture that

$$
|G(a)| \leq \min \left\{(k-1) p^{1 / 2},(1+\eta)(2 k p \log k p)^{1 / 2}\right\},
$$

where $\eta \rightarrow 0$ as $k$ and $p / k$ tend to infinity. Indeed one expects that this hypothetical upper bound would be best possible.

The estimate (1) is fairly sharp if $k$ is small in comparison with $p$, but as soon as $k \gg \sqrt{p}$ it becomes worse than the trivial bound. This is a universal problem when one applies Weil's method, (or indeed Deligne's, in the case of multiple exponential sums): For large degree the bound obtained is trivial.

For values of $k$ of intermediate size remarkable progress was made by Shparlinski [6], who established the bound

$$
G(a) \ll k^{7 / 12} p^{2 / 3},
$$

thereby improving the previous results for $p^{2 / 5} \leq k \leq p^{4 / 7}$. Moreover Konyagin and Shparlinski later showed, in unpublished work, that

$$
G(a) \ll k^{1 / 3} p^{19 / 24},
$$

which improves the three earlier bounds for $p^{1 / 2} \leq k \leq p^{5 / 8}$. Both the results (3) and (4) were subsequently found independently by Heath-Brown (unpublished).

Shparlinski reduces the problem of estimating $G(a)$ to that of bounding the number of solutions to a congruence

$$
x^{k}+y^{k} \equiv n(\bmod p) .
$$

This problem is tackled via a theorem of Garcia and Voloch [1]. Heath-Brown's approach is very similar, but the method of Stepanov [7] is used to handle (5). The proof of Garcia and Voloch's estimate has in fact strong parallels with Stepanov's method.

It should also be mentioned that large values of $k$ have been treated by Konyagin [3], who shows that for any $\varepsilon>0$ there is a positive constant $c_{\varepsilon}$ for which

$$
|G(a)| \leq p\left(1-\frac{c_{\varepsilon}}{(\log k)^{1+\varepsilon}}\right)
$$


for $k \geq 2$ and

$$
p \geq \frac{k \log k}{(\log \log k)^{1-\varepsilon}} .
$$

Here we have corrected an unfortunate misprint in the English translation of Konyagin's paper, which led to its being quoted incorrectly in both Zentralblatt, (820:11048) and Math. Reviews, (96e:11122). Although the improvement over the trivial bound is extremely small, there are important consequences for Waring's problem modulo $p$, as Konyagin describes.

In the present paper we improve the application of Stepanov's method to bound the number of solutions of (5) for several different values of $n$ simultaneously. This enables us to establish the following improvement of (3).

Theorem 1 For $p \nmid$ a we have

$$
G(a) \ll\left\{\begin{array}{cc}
k p^{1 / 2}, & 1 \leq k \leq p^{1 / 3}, \\
k^{5 / 8} p^{5 / 8}, & p^{1 / 3}<k \leq p^{1 / 2}, \\
k^{3 / 8} p^{3 / 4}, & p^{1 / 2}<k \leq p^{2 / 3}, \\
p, & p^{2 / 3}<k<p .
\end{array}\right.
$$

The trivial bound and the estimate (1) are therefore both superseded for $p^{1 / 3} \ll$ $k \ll p^{2 / 3}$.

For many years it was an open problem to show that Heilbronn's sum satisfies $H_{p}(a)=o(p)$ as $p \rightarrow \infty$. Recently Heath-Brown [2] was able to establish the bound

$$
H_{p}(a) \ll p^{11 / 12} .
$$

The proof used Stepanov's method to bound the number of solutions of the congruence

$$
f(x) \equiv u(\bmod p)
$$

where

$$
f(X)=X+\frac{X^{2}}{2}+\frac{X^{3}}{3}+\ldots+\frac{X^{p-1}}{p-1},
$$

thereby re-discovering a result of Mit'kin [4]. Our new variant of Stepanov's method can be applied here too, yielding the following improved estimate.

Theorem 2 We have

$$
\sum_{r=1}^{p}\left|H_{p}(a+r p)\right|^{4} \ll p^{7 / 2}
$$

and hence

$$
H_{p}(a) \ll p^{7 / 8}
$$

for $p \nmid a$. 
As a corollary, we have a new bound for incomplete Heilbronn sums.

Corollary If $p$ is a prime and $p \nmid$ a then

$$
\sum_{\substack{M<n \leq M+N \\ p \nmid n}} e\left(\frac{a n^{p}}{p^{2}}\right) \ll p^{5 / 8} N^{1 / 4},
$$

uniformly in a, for all $M$ and for all $N \leq p$.

This may be compared with the corresponding result of Heath-Brown [2], in which the bound was $O\left(p^{11 / 12}\right)$. The new result is non-trivial for $N \gg p^{5 / 6}$.

The proofs of our theorems begin with some straightforward manipulation, leading to the following results.

Lemma 1 Let $h=(p-1) / k$ and set

$$
\begin{gathered}
\mu_{h}=\left\{x \in \mathbb{Z}_{p}: x^{h}=1\right\} \\
\mathcal{A}(h)=\left\{\left(x_{1}, x_{2}, x_{3}, x_{4}\right) \in \mu_{h}^{4}: x_{1}+x_{2}=x_{3}+x_{4}\right\} .
\end{gathered}
$$

Then

$$
G(a) \ll k^{5 / 4}(\# \mathcal{A}(h))^{1 / 4},
$$

and

$$
G(a) \ll p^{1 / 8} k(\# \mathcal{A}(h))^{1 / 4}
$$

Lemma 2 Let

$$
f(X)=X+\frac{X^{2}}{2}+\frac{X^{3}}{3}+\ldots+\frac{X^{p-1}}{p-1} \in \mathbb{Z}_{p}
$$

and let

$$
\mathcal{B}=\left\{\left(x_{1}, x_{2}\right) \in \mathbb{Z}_{p}^{2}: f\left(x_{1}\right)=f\left(x_{2}\right)\right\}
$$

Then

$$
\sum_{r}^{p}\left|H_{p}(a+r p)\right|^{4} \ll p^{3}+p^{2} \# \mathcal{B} .
$$

By applying our new variant of Stepanov's method we shall establish the following bounds for $\# \mathcal{A}(h)$ and $\# \mathcal{B}$, from which Theorems 1 and 2 immediately follow.

Lemma 3 For any $h<p^{2 / 3}$ we have $\# \mathcal{A}(h) \ll h^{5 / 2}$.

Lemma 4 We have $\# \mathcal{B} \ll p^{3 / 2}$. 
The nature of our improvement in the application of Stepanov's method is clearest when one compares Lemma 4 of Heath-Brown [2], with our Lemma 7. If we define

$$
\mathcal{F}(u)=\left\{x \in \mathbb{Z}_{p}: f(x)=u\right\}
$$

then, in the notation of the current paper, the former result states that

$$
\# \mathcal{F}(u) \ll p^{2 / 3}
$$

for any $u \in \mathbb{Z}_{p}$, while our Lemma 7 shows that

$$
\sum_{u \in U} \# \mathcal{F}(u) \ll p^{2 / 3}(\# U)^{2 / 3}
$$

for any $U \subseteq \mathbb{Z}_{p}$.

\section{Proof of Lemmas 1 and 2}

In this section we shall prove Lemmas 1 and 2 . We begin by writing

$$
G_{0}(a)=\sum_{n=1}^{p-1} e_{p}\left(a n^{k}\right)
$$

so that $G(a)=1+G_{0}(a)$. Then

$$
G_{0}(a)=G_{0}\left(a m^{k}\right) \text { for } p \nmid m .
$$

It follows that

$$
(p-1)\left|G_{0}(a)\right|^{4}=\sum_{m=1}^{p-1}\left|G_{0}\left(a m^{k}\right)\right|^{4} \leq k \sum_{n=1}^{p}\left|G_{0}(n)\right|^{4},
$$

since each value of $n$ arises either $k$ times or not at all. We therefore see that

$$
\begin{aligned}
h\left|G_{0}(a)\right|^{4} & \leq \sum_{m_{1}, \ldots, m_{4}=1}^{p-1} \sum_{n=1}^{p} e_{p}\left(\left(m_{1}^{k}+m_{2}^{k}-m_{3}^{k}-m_{4}^{k}\right) n\right) \\
& =p \#\left\{\left(m_{1}, \ldots, m_{4}\right): m_{1}^{k}+m_{2}^{k} \equiv m_{3}^{k}+m_{4}^{k}(\bmod p)\right\} \\
& =p k^{4} \# \mathcal{A}(h),
\end{aligned}
$$

and (6) follows.

To derive $(7)$ we note that

$$
(p-1)\left|G_{0}(a)\right|^{2}=\sum_{m=1}^{p-1}\left|G_{0}\left(a m^{k}\right)\right|^{2}
$$




$$
\begin{aligned}
& =\sum_{n_{1}, n_{2}=1}^{p-1} \sum_{m=1}^{p-1} e_{p}\left(a\left(n_{1}^{k}-n_{2}^{k}\right) m^{k}\right) \\
& =\sum_{b=1}^{p} N(b) G_{0}(a b)
\end{aligned}
$$

where

$$
N(b)=\#\left\{\left(n_{1}, n_{2}\right): 1 \leq n_{1}, n_{2} \leq p-1, n_{1}^{k}-n_{2}^{k} \equiv b(\bmod p)\right\} .
$$

We may now apply Hölder's inequality, whence

$$
(p-1)^{4}\left|G_{0}(a)\right|^{8} \leq\left\{\sum_{b=1}^{p} N(b)^{2}\right\}\left\{\sum_{b=1}^{p} N(b)\right\}^{2}\left\{\sum_{b=1}^{p}\left|G_{0}(a b)\right|^{4}\right\} .
$$

As above, the final sum on the right is $p k^{4} \# \mathcal{A}(h)$. We may therefore conclude that

$$
(p-1)^{4}\left|G_{0}(a)\right|^{8} \ll p k^{4}(\# \mathcal{A}(h))\left\{\sum_{b=1}^{p} N(b)^{2}\right\}\left\{\sum_{b=1}^{p} N(b)\right\}^{2} .
$$

In order to estimate the terms involving the function $N(b)$, we recall that $h=(p-1) / k$, and observe that the congruence $n^{k} \equiv s(\bmod p)$ has no solutions unless $s^{h} \equiv 1(\bmod p)$, in which case there are exactly $k$ solutions. It therefore follows that $N(b)=k^{2} M(b)$, where

$$
M(b)=\#\left\{\left(x_{1}, x_{2}\right) \in \mu_{h}^{2}: x_{1}-x_{2}=b\right\} .
$$

We trivially have

$$
\sum_{b=1}^{p} M(b)^{2}=\mathcal{A}(h)
$$

whence

$$
\sum_{b=1}^{p} N(b)^{2}=k^{4} \mathcal{A}(h)
$$

Moreover it is clear that

$$
\sum_{b=1}^{p} N(b)=(p-1)^{2} .
$$

If we now insert these formulae into (8) we see that the estimate (7) follows immediately.

The proof of Lemma 2 is similar to that of (6). We write

$$
H_{0}(a)=\sum_{n=1}^{p-1} e\left(\frac{a n^{p}}{p^{2}}\right)
$$


so that $H(a)=1+H_{0}(a)$. Then

$$
H_{0}(a)=H_{0}\left(a m^{p}\right) \text { for } p \nmid m .
$$

It follows that

$$
(p-1) \sum_{r=1}^{p}\left|H_{0}(a+r p)\right|^{4}=\sum_{r=1}^{p} \sum_{m=1}^{p-1}\left|H_{0}\left((a+r p) m^{p}\right)\right|^{4} \leq \sum_{n=1}^{p^{2}}\left|H_{0}(n)\right|^{4},
$$

since each value of $n$ arises at most once. (Indeed each value with $p \nmid n$ arises exactly once.) We therefore see that

$$
\begin{aligned}
&(p-1) \sum_{r=1}^{p} \mid\left.H_{0}(a+r p)\right|^{4} \leq \sum_{m_{1}, \ldots, m_{4}=1}^{p-1} \sum_{n=1}^{p^{2}} e_{p^{2}}\left(\left(m_{1}^{p}+m_{2}^{p}-m_{3}^{p}-m_{4}^{p}\right) n\right) \\
&=p^{2} \#\left\{1 \leq m_{1}, \ldots, m_{4} \leq p-1: m_{1}^{p}+m_{2}^{p} \equiv m_{3}^{p}+m_{4}^{p}\left(\bmod p^{2}\right)\right\} .
\end{aligned}
$$

Here we must have $m_{1}+m_{2} \equiv m_{3}+m_{4}(\bmod p)$. Thus, if we write

$$
m_{1}-m_{3} \equiv b(\bmod p)
$$

we also have $m_{4}-m_{2} \equiv b(\bmod p)$. The case $p \mid b$ now contributes $(p-1)^{2}$ solutions of the congruence. When $p \nmid b$ we write $m_{1} \equiv v_{1} b(\bmod p)$, so that $m_{3} \equiv\left(v_{1}-1\right) b(\bmod p)$. Thus

$$
m_{1}^{p}-m_{3}^{p} \equiv\left(v_{1}^{p}-\left(v_{1}-1\right)^{p}\right) b^{p}\left(\bmod p^{2}\right) .
$$

In the same way we find that

$$
m_{4}^{p}-m_{2}^{p} \equiv\left(v_{2}^{p}-\left(v_{2}-1\right)^{p}\right) b^{p}\left(\bmod p^{2}\right),
$$

where $m_{4} \equiv v_{2} b(\bmod p)$.

The congruence $m_{1}^{p}+m_{2}^{p} \equiv m_{3}^{p}+m_{4}^{p}\left(\bmod p^{2}\right)$ now becomes

$$
\left(v_{1}^{p}-\left(v_{1}-1\right)^{p}\right) b^{p} \equiv\left(v_{2}^{p}-\left(v_{2}-1\right)^{p}\right) b^{p}\left(\bmod p^{2}\right) .
$$

There are $p-1$ choices for $b$, and for each such value we will have

$$
v_{1}^{p}-\left(v_{1}-1\right)^{p} \equiv v_{2}^{p}-\left(v_{2}-1\right)^{p}\left(\bmod p^{2}\right) .
$$

Since

$$
v^{p}-(v-1)^{p}=\sum_{l=1}^{p}(-1)^{l-1} v^{p-l}\left(\begin{array}{c}
p \\
l
\end{array}\right) \equiv 1-p f(v)\left(\bmod p^{2}\right),
$$

it now follows that

$$
\begin{aligned}
& \#\left\{1 \leq m_{1}, \ldots, m_{4} \leq p-1: m_{1}^{p}+m_{2}^{p} \equiv m_{3}^{p}+m_{4}^{p}\left(\bmod p^{2}\right)\right\} \\
& \quad \leq(p-1)^{2}+(p-1) \#\left\{1 \leq v_{1}, v_{2} \leq p-1: f\left(v_{1}\right) \equiv f\left(v_{2}\right)(\bmod p)\right\},
\end{aligned}
$$

whence

$$
(p-1) \sum_{r=1}^{p}\left|H_{0}(a+r p)\right|^{4} \leq p^{2}\left\{(p-1)^{2}+(p-1) \# \mathcal{B}\right\}
$$

which suffices for Lemma 2. 


\section{Stepanov's Method}

We shall begin by considering $\# \mathcal{A}(h)$. For each $u \in \mathbb{Z}_{p}$ we write

$$
\mathcal{C}(u)=\left\{x \in \mu_{h}: x-u \in \mu_{h}\right\},
$$

so that $\# \mathcal{C}(0)=h$ and

$$
\begin{aligned}
\# \mathcal{A}(h) & =\sum_{u \in \mathbb{Z}_{p}}(\# \mathcal{C}(u))^{2} \\
& =h^{2}+\sum_{u \neq 0}(\# \mathcal{C}(u))^{2} \\
& =h^{2}+h \sum_{u}{ }^{*}(\# \mathcal{C}(u))^{2}
\end{aligned}
$$

where $\Sigma^{*}$ indicates that $u$ runs over distinct coset representatives of $\mu_{h}$ in $\mathbb{Z}_{p}^{\times}$.

In the same way we have

$$
\begin{aligned}
\left\{\# \mu_{h}\right\}^{2} & =\sum_{u \in \mathbb{Z}_{p}} \# \mathcal{C}(u) \\
& =h+\sum_{u \neq 0} \# \mathcal{C}(u) \\
& =h+h \sum_{u}{ }^{*} \# \mathcal{C}(u),
\end{aligned}
$$

whence

$$
\sum_{u}^{*} \# \mathcal{C}(u)=h-1
$$

We now take an arbitrary set $U$ of elements $u$ from distinct cosets of $\mathbb{Z}_{p}^{\times}$, and write

$$
\mathcal{D}(u)=u^{-1} \mathcal{C}(u)=\#\left\{y \in \mathbb{Z}_{p}: u y \in \mu_{h}, u y-u \in \mu_{h}\right\},
$$

and

$$
\mathcal{E}=\bigcup_{u \in U} \mathcal{D}(u)
$$

Thus $\# \mathcal{D}(u)=\# \mathcal{C}(u)$, and since the sets $\mathcal{D}(u)$ are disjoint we deduce that

$$
\# \mathcal{E}=\sum_{u \in U} \# \mathcal{C}(u) .
$$

Our aim is to prove the following bound for $\# \mathcal{E}$. 
Lemma 5 Let $\# U=T \geq 1$. Then

$$
\# \mathcal{E} \ll(h T)^{2 / 3}
$$

providing that $h^{4} T<p^{3}$.

We begin our application of Stepanov's method by taking a polynomial $\Phi(X, Y, Z) \in \mathbb{Z}_{p}[X, Y, Z]$, for which

$$
\operatorname{deg}_{X} \Phi<A, \operatorname{deg}_{Y} \Phi<B, \operatorname{deg}_{Z} \Phi<B,
$$

and arranging that the polynomial

$$
\Psi(X)=\Phi\left(X, X^{h},(X-1)^{h}\right)
$$

has a zero of order at least $D$, say, at each point $x \in \mathcal{E}$. We will therefore be able to conclude that $D \# \mathcal{E} \leq \operatorname{deg} \Psi(X)$, providing that $\Psi$ does not vanish identically. We note that

$$
\operatorname{deg} \Psi \leq\left(\operatorname{deg}_{X} \Phi\right)+h\left(\operatorname{deg}_{Y} \Phi\right)+h\left(\operatorname{deg}_{Z} \Phi\right)<A+2 h B,
$$

whence

$$
D \# \mathcal{E} \ll A+h B,
$$

providing that $\Psi$ does not vanish.

In order for $\Psi$ to have a zero of multiplicity at least $D$ at a point $x$ we need

$$
\left.\left(\frac{d}{d X}\right)^{n} \Psi(X)\right|_{X=x}=0 \quad \text { for } n<D .
$$

Since $x \neq 0,1$ for $x \in \mathcal{E}$, this will be equivalent to

$$
\left.\{X(X-1)\}^{n}\left(\frac{d}{d X}\right)^{n} \Psi(X)\right|_{X=x}=0 .
$$

We now observe that

$$
\begin{aligned}
X^{m}\left(\frac{d}{d X}\right)^{m} X^{a} & =\frac{a !}{(a-m) !} X^{a}, \\
X^{m} \frac{d^{m}}{d X^{m}} X^{h b} & =\frac{(h b) !}{(h b-m) !} X^{h b},
\end{aligned}
$$

and

$$
(X-1)^{m}\left(\frac{d}{d X}\right)^{m}(X-1)^{h c}=\frac{(h c) !}{(h c-m) !}(X-1)^{h c} .
$$

It follows that

$$
\{X(X-1)\}^{n}\left(\frac{d}{d X}\right)^{n} X^{a} X^{h b}(X-1)^{h c}=P_{n, a, b, c}(X) X^{h b}(X-1)^{h c}
$$


where $P_{n, a, b, c}(X)$ either vanishes or is a polynomial of degree $n+a$. We therefore deduce that

$$
\left.\{X(X-1)\}^{n}\left(\frac{d}{d X}\right)^{n} X^{a} X^{h b}(X-1)^{h c}\right|_{X=x}=u^{-h b-h c} P_{n, a, b, c}(x)
$$

for any $x \in \mathcal{D}(u)$. Here we use the fact that $x^{h}=(x-1)^{h}=u^{-h}$ for such $x$.

We now write

$$
\Phi(X, Y, Z)=\sum_{a, b, c} \lambda_{a, b, c} X^{a} Y^{b} Z^{c}
$$

and

$$
P_{n, u}(X)=\sum_{a, b, c} \lambda_{a, b, c} u^{-h b-h c} P_{n, a, b, c}(X),
$$

so that $\operatorname{deg} P_{n, u}(X)<A+n$ and

$$
\left.\{X(X-1)\}^{n}\left(\frac{d}{d X}\right)^{n} \Phi\left(X, X^{h},(X-1)^{h}\right)\right|_{X=x}=P_{n, u}(x)
$$

for any $x$ in $\mathcal{D}(u)$. We shall arrange, by appropriate choice of the coefficients $\lambda_{a, b, c}$, that $P_{n, u}(X)$ vanishes identically for $n<D$, for all $u \in U$. This will ensure that (12) holds for $x \in \mathcal{E}$. Each of the polynomials $P_{n, u}(X)$ has at most $A+n \leq A+D$ coefficients, which are linear forms in the original $\lambda_{a, b, c}$. Thus if

$$
D(A+D) T<A B^{2},
$$

there will be a set of coefficents $\lambda_{a, b, c}$, not all zero, for which the polynomials $P_{n, u}(X)$ vanish for all $n<D$ and all $u \in U$.

We must now consider whether $\Phi\left(X, X^{h},(X-1)^{h}\right)$ can vanish if $\Phi(X, Y, Z)$ does not. We shall write

$$
\Phi(X, Y, Z)=\sum_{c} \Phi_{c}(X, Y) Z^{c}
$$

and take $c_{0}$ to be the smallest value of $c$ for which $\Phi_{c}(X, Y)$ is not identically zero. It follows that

$$
\Phi\left(X, X^{h},(X-1)^{h}\right)=(X-1)^{h c_{0}} \sum_{c_{0} \leq c<B} \Phi_{c}\left(X, X^{h}\right)(X-1)^{h\left(c-c_{0}\right)},
$$

so that if $\Phi\left(X, X^{h},(X-1)^{h}\right)$ is identically zero we must have

$$
\Phi_{c_{0}}\left(X, X^{h}\right) \equiv 0\left(\bmod (X-1)^{h}\right) .
$$

At the end of this section we shall establish the following result.

Lemma 6 Let $P(X) \in \mathbb{Z}_{p}[X]$ be a sum of $N \geq 1$ distinct monomials. Suppose further that $\operatorname{deg}(P)<p$. Then $(X-1)^{N}$ cannot divide $P(X)$. 
Lemma 6 shows that (14) is impossible, providing that

$$
A B \leq h \text { and } A+h B<p .
$$

We now choose our parameters $A$ and $B$ by taking

$$
A=\left[\frac{1}{2} h^{2 / 3} T^{-1 / 3}\right] \text { and } B=\left[\frac{1}{2} h^{1 / 3} T^{1 / 3}\right] .
$$

These will produce positive integers satisfying (15), providing that $h^{2} \geq 8 T$ and $h^{4} T<p^{3}$. Moreover there will then be an integer $T$ for which (13) holds, in the range $h^{2 / 3} T^{-1 / 3} \ll D \ll h^{2 / 3} T^{-1 / 3}$. The estimate (11) therefore produces

$$
\# \mathcal{E} \ll h B / D \ll(h T)^{2 / 3}
$$

as required. Of course, if $T \gg h^{2}$, then the bound (10) yields

$$
\# \mathcal{E} \ll h \ll(h T)^{2 / 3},
$$

and Lemma 5 is trivial.

We turn now to the argument required for Lemma 4. This will be an adaption of that given by Heath-Brown [2], along the lines used above. Thus we write

$$
\mathcal{F}(u)=\left\{x \in \mathbb{Z}_{p}: f(x)=u\right\}
$$

so that

$$
\# \mathcal{B}=\sum_{u \in \mathbb{Z}_{p}}(\# \mathcal{F}(u))^{2}
$$

and

$$
\sum_{u \in \mathbb{Z}_{p}} \# \mathcal{F}(u)=p
$$

Moreover we set

$$
\mathcal{G}=\bigcup_{u \in U} \mathcal{F}(u)
$$

where $U$ is an arbitrary set of $T$ elements $u \in \mathbb{Z}_{p}$. In analogy to Lemma 5 we aim to prove the following bound.

Lemma 7 Let $\# U=T \geq 1$. Then

$$
\# \mathcal{G} \ll(p T)^{2 / 3} .
$$

We begin by choosing $\Phi(X, Y, Z) \in \mathbb{Z}_{p}[X, Y, Z]$, with

$$
\operatorname{deg}_{X} \Phi<A, \operatorname{deg}_{Y} \Phi<B, \operatorname{deg}_{Z} \Phi<C .
$$

We shall arrange that the polynomial

$$
\Psi(X)=\Phi\left(X, f(X), X^{p}\right)
$$


has a zero of order at least $D$, say, at each point $x \in \mathcal{G}$. We will then be able to deduce that $D \# \mathcal{G} \leq \operatorname{deg} \Psi(X)$, providing that $\Psi$ does not vanish identically. We note that

$$
\operatorname{deg} \Psi \leq\left(\operatorname{deg}_{X} \Phi\right)+(p-1)\left(\operatorname{deg}_{Y} \Phi\right)+p\left(\operatorname{deg}_{Z} \Phi\right)<A+p(B+C),
$$

whence

$$
D \# \mathcal{G} \ll A+p(B+C),
$$

providing that $\Psi$ does not vanish.

Following the argument of $[2 ; \S \S 3 \& 4]$ this can be achieved by making certain polynomials $P_{n, u}(X)$ of degree less than $A+2 D+C$ vanish identically, for all $n<D$ and each $u \in U$. The coefficients of these polynomials are linear forms in the coefficents of the original function $\Phi$, so that it suffices to have

$$
D(A+2 D+C) T<A B C .
$$

Moreover Lemma 3 of [2] shows that $\Psi$ will not vanish identically, providing that

$$
A B \leq p
$$

We therefore choose

$$
A=\left[p^{2 / 3} T^{-1 / 3}\right], \quad B=C=\left[p^{1 / 3} T^{1 / 3}\right],
$$

which are clearly admissable, since $T=\# U \leq p$. Moreover we may take

$$
D=\left[p^{2 / 3} T^{-1 / 3} / 16\right],
$$

which is also satisfactory, if $p$ is large enough. It then follows from (18) that

$$
\# \mathcal{G} \ll p^{2 / 3} T^{2 / 3}
$$

as required.

It remains to establish Lemma 6 . This will be achieved by induction on $N$. The case $N=1$ is trivial. Now suppose that $N>1$, and let

$$
P(X)=\sum_{l} c_{l} X^{l}
$$

where $l$ runs over $N$ distinct values. Then

$$
X P^{\prime}(X)-l_{0} P(X)=\sum_{l} c_{l}\left(l-l_{0}\right) X^{l} .
$$

Now, on choosing $l_{0}$ to be, say, the degree of the highest order term in $P(X)$, we produce a polynomial containing exactly $N-1$ terms. We then see that $(X-1)^{N}$ cannot divide $P(X)$, for otherwise $(X-1)^{N-1}$ would divide $X P^{\prime}(X)-l_{0} P(X)$, contrary to our induction hypothesis. This completes the proof of Lemma 6 . 


\section{Deduction of Lemmas 3 and 4}

We shall now use Lemma 5 , in conjunction with (9) and (10), to bound $\# \mathcal{A}(h)$. Since we are assuming that $h \leq p^{2 / 3}$ it is automatic that

$$
h^{4} T \leq h^{4} k=h^{3}(p-1)<p^{3} .
$$

We number the coset representatives $u$ as $u_{i}, 1 \leq i \leq k$, in such a way that

$$
\# \mathcal{C}\left(u_{1}\right) \geq \# \mathcal{C}\left(u_{2}\right) \geq \ldots
$$

If we now take $U$ to be the set of $u_{i}$ for $i \leq T$ then Lemma 5 shows that

$$
T \# \mathcal{C}\left(u_{T}\right) \leq \# \mathcal{E} \ll(h T)^{2 / 3}
$$

for any $T$. Hence

$$
\sum_{N / 2<T \leq N}\left(\# \mathcal{C}\left(u_{T}\right)\right)^{2} \ll N\left(h^{2 / 3} N^{-1 / 3}\right)^{2}=h^{4 / 3} N^{1 / 3} .
$$

Alternatively we may use (10), which yields

$$
\sum_{N / 2<T \leq N}\left(\# \mathcal{C}\left(u_{T)}\right)^{2} \ll h^{2 / 3} N^{-1 / 3}(h-1) \ll h^{5 / 3} N^{-1 / 3} .\right.
$$

If we now sum over $N=1,2,4,8, \ldots$, using the first bound for $N \leq h^{1 / 2}$ and the second estimate otherwise, we find that

$$
\sum_{u}^{*}(\# \mathcal{C}(u))^{2} \ll h^{3 / 2},
$$

so that Lemma 3 follows from (9).

The deduction of Lemma 4 from (16), (17) and Lemma 7 is, of course, completely analogous.

\section{The Corollary to Theorem 2}

As in Heath-Brown [2], the standard procedure for completing an incomplete exponential sum yields

$$
\begin{aligned}
\sum_{\substack{M<n \leq M+N \\
p \nmid n}} e\left(\frac{a n^{p}}{p^{2}}\right) & =p^{-1} \sum_{r=1}^{p} \sum_{s=1}^{p} e\left(\frac{a s^{p}}{p^{2}}\right) \sum_{M<n \leq M+N} e\left(\frac{r(s-n)}{p}\right) \\
& \ll p^{-1} \sum_{r=1}^{p} \min \left\{N, \frac{1}{\|r / p\|}\right\}\left|\sum_{s=1}^{p} e\left(\frac{a s^{p}}{p^{2}}\right) e\left(\frac{r s}{p}\right)\right|,
\end{aligned}
$$


on using the estimates

$$
\sum_{M<n \leq M+N} e\left(\frac{-r n}{p}\right) \ll\left\{\begin{array}{cc}
N, & \text { any } r \\
\frac{1}{\|r / p\|}, & p \nmid r .
\end{array}\right.
$$

However, since $s \equiv s^{p}(\bmod p)$, we have

$$
e\left(\frac{a s^{p}}{p^{2}}\right) e\left(\frac{r s}{p}\right)=e\left(\frac{(a+r p) s^{p}}{p^{2}}\right),
$$

so that

$$
\sum_{s=1}^{p} e\left(\frac{a s^{p}}{p^{2}}\right) e\left(\frac{r s}{p}\right)=H(a+r p)
$$

and hence

$$
\sum_{\substack{M<n \leq M+N \\ p \nmid n}} e\left(\frac{a n^{p}}{p^{2}}\right) \ll p^{-1} \sum_{r=1}^{p} \min \left\{N, \frac{1}{\|r / p\|}\right\}|H(a+r p)| .
$$

We may now apply Hölder's inequality, whence

$$
\begin{aligned}
& \sum_{\substack{M<n \leq M+N \\
p \nmid n}} e\left(\frac{a n^{p}}{p^{2}}\right) \\
& \ll p^{-1}\left\{\sum_{r=1}^{p} \min \left\{N, \frac{1}{\|r / p\|}\right\}^{4 / 3}\right\}^{3 / 4}\left\{\sum_{r=1}^{p}|H(a+r p)|^{4}\right\}^{1 / 4} \\
& \ll p^{-1 / 8}\left\{\sum_{r=1}^{p} \min \left\{N, \frac{1}{\|r / p\|}\right\}^{4 / 3}\right\}^{3 / 4},
\end{aligned}
$$

by Theorem 2 . Since $N \leq p$ and

$$
\sum_{r=1}^{p} \min \left\{N, \frac{1}{\|r / p\|}\right\}^{4 / 3} \ll p N^{1 / 3}
$$

we deduce that

$$
\sum_{\substack{M<n \leq M+N \\ p \nmid n}} e\left(\frac{a n^{p}}{p^{2}}\right) \ll p^{5 / 8} N^{1 / 4}
$$

as claimed. 


\section{Acknowledgement}

The second author was supported by Grants 96-01-00378 from the Russian Foundation for Basic Research and 96-15-96072 from the Programme of Development of Scientific Schools.

\section{References}

[1] A. Garcia and J. F. Voloch, Fermat Curves Over Finite Fields, J. Number Theory, 30 (1988), 345-356.

[2] D.R. Heath-Brown, An estimate for Heilbronn's exponential sum, Analytic number theory: Proceedings of a conference in honor of Heini Halberstam, (Birkhäuser, Boston, 1996), 451-463.

[3] S.V. Konyagin, On estimates of Gaussian sums and Waring's problem for a prime modulus, Proc. Steklov Inst. Math., 198 (1994), 105-117.

[4] D.A. Mit'kin, An estimate for the number of roots of some comparisons by the Stepanov method, Mat. Zametki, 51 (1992), 52-58, 157. (Translated as Math. Notes, 51 (1992), 565-570.)

[5] H.L. Montgomery, R.C. Vaughan, and T.D. Wooley, Some remarks on Gauss sums associated with $k$ th powers, Math. Proc. Camb. Phil. Soc., 118 (1995), 21-33.

[6] I. E. Shparlinski, On Bounds of Gaussian Sums, Mat. Zametki, 50 (1991), $122-130$.

[7] S.A. Stepanov, The number of points of a hyperelliptic curve over a prime field, Izv. Akad. Nauk SSSR Ser. Mat., 33 (1969), 1171-1181. 